\title{
MicroRNAs, Small Gene Regulators with High Therapeutic Promise in Oncology
}

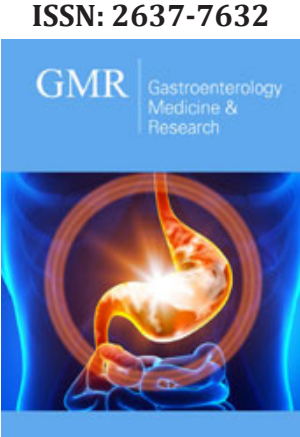

*Corresponding author: Christophe $\mathrm{F}$ Grosset, miRCaDe Team (microRNA in Cancer and Development), 146, Rue Léo Saignat, 33076 Bordeaux Cedex, France

Submission: 望 July 5, 2019

Published: 恝July 18, 2019

Volume 3 - Issue 3

How to cite this article: Véronique Trézéguet, Christophe F Grosset. MicroRNAs, Small Gene Regulators with High Therapeutic Promise in Oncology Gastro Med Res. 3(3). GMR.000565. 2019. DOI: $10.31031 /$ GMR.2019.03.000565

Copyright@ Christophe F Grosset, This article is distributed under the terms of the Creative Commons Attribution 4.0 International License, which permits unrestricted use and redistribution provided that the original author and source are credited.

\author{
Véronique Trézéguet and Christophe F Grosset*
}

miRCaDe team, Bordeaux, France

\begin{abstract}
Since their discovery in the mid-1990s, microRNAs (miRNAs) have captured the attention of the scientific community by their ability to post-transcriptionally and subtly regulate a large but still poorly defined set of gene targets and to control most cellular and molecular processes, making them central effectors of gene reprogramming and cell functioning under both normal and pathophysiological conditions. In this mini-review we summarize step by step the molecular mechanisms involved in miRNA biogenesis and mRNA regulation, the latter depending directly on the duration of the miRNA-mediated translational arrest and the integrity of the transcript extremities. We also discuss the nomenclature of miRNAs, the pairing rules between a miRNA and its target through the seed sequence, and the remarkable complexity of the interactions between thousands of genes and miRNAs in a cell at the origin of a regulatory and communicative network that intriguingly evokes the neuronal interconnections occurring in the brain. We recall that miRNAs are not only intracellular gene regulators but also trans-communicators capable of operating remotely from their production site in blood-circulating macrovesicles or exosomes. We stress their role in tissue carcinogenesis and their use as antitumoral agents with two examples of miRNAs that have reached the clinic but with various successes. Finally, we conclude by stating that despites the many obstacles to be overcome, miRNAs remain major candidates for therapeutic use in cancer and therefore, the scientific community should continue its efforts to pursue investigations in the field of miRNA to develop intelligent, safe and controllable systems and vectors for miRNA delivery.
\end{abstract}

\section{Introduction}

MicroRNAs (miRNAs) are small non-coding RNAs of 18 to 24 nucleotides in length encountered in animals, plants and some viruses. Their main biological function is to finely and concomitantly tune expression of multiple genes in a spatial and temporal manner with the aim at strictly controlling the protein amount encoded by these genes. miRNAs are thus key players in cellular reprograming, molecular signaling and physiological processes such as cell homeostasis, death or motility, but their deregulation is also central in many pathologies such as cancer.

\section{miRNA biogenesis and molecular mechanisms}

The canonical pathway of miRNA biogenesis starts in the nucleus with the transcription of the miRNA gene by RNA polymerase II, which results in the production of a primary transcript (Pri-miRNA) (Figure 1). This Pri-miRNA is polyadenylated, capped and contains secondary hairpin structures at specific positions. These structures are recognized by a protein complex called the "microprocessor" and composed of Drosha and DGCR8 proteins (DiGeorge syndrome critical region 8). Cleavage by Drosha generates the precursor miRNA (Pre-miRNA), which is about 70-nucleotide long. After export to the cytoplasm, a second protein complex, composed of Dicer and its cofactor TRBP (TAR RNA-binding protein), binds the Pre-miRNA. Cleavage by Dicer leads to a mature and imperfectly complementary miRNAmiRNA duplex. This duplex is then processed by one of the 4 Argonaut proteins that separate the two RNA strands so that one is selected to generate the effector complex called "miRNAinduced silencing complex" (miRISC). When either of the two strands of the mature miRNAmiRNA duplex is loaded into miRISC without any particular preference, the miRNA generated from the 5' arm of the Pre-miRNA is called "miR-XXX-5p" (ex: miR-1271-5p) and that from the 3' arm is called "miR-XXX-3p" (ex: miR-1271-3p). When one particular strand of the duplex is loaded almost exclusively (usually the 5 ' arm of the Pre-miRNA), it is called "miR-XXX" without extension (e. g. miR-4510). Once miRISC is loaded with a single-stranded miRNA, it pairs its target transcript (most often a messenger RNA, mRNA) to activate and regulate the target expression through a post-transcriptional mechanism involving both mRNA translation and degradation machineries. 
The latest studies in this field evidenced a complex and sequential molecular process (Figure 1) that begins with the repression of the mRNA translation which, if lasting, is followed by the deadenylation of the mRNA at the 3' end by the PAN2-PAN3 and CCR4-NOT complexes and the cap removal at the 5' end by the DCP1-DCP2 complex. Thus, the mRNA deprived of its protective extremities is rapidly degraded by specific exonucleases (e. g. XRN1) [1]. This process is highly regulated and depends on many factors such as the stability of the miRNA:target pair, the cell type, the molecular content of cells and environmental factors (stimulus, phosphorylation, sumoylation) [2].

In mammals, miRNAs act on mRNAs by targeting preferentially the 3'-untranslated region (3'-UTR), then the coding region and finally the 5'-untranslated region (5'-UTR). The pairing rules between a miRNA and its target are multifactorial and depend mostly on the position and accessibility of the miRNA site within the mRNA but also on the nature of the surrounding sequence, the RNA secondary and tertiary structures and the associated RNAbinding proteins. In the case of a miRNA site located in the 3'-UTR, the miRNA generally interacts with the mRNA through its $5^{\prime}$ part, called the "seed", which includes nucleotides 2 to 7. This pairing is often reinforced by complementary pairing with the target, in particular, the miRNA base 8 and nucleotides 13 and 16 on the 3' side [3]. An adenosine located in the 3'-UTR region of the target, immediately downstream of the miRNA site, also promotes miRNA action by stabilizing the interactions between the mRNA and miRISC [2]. Finally, a recent paper by Kai Zhang and coworkers reported an alternative miRNA-target recognition mechanism for mRNA with miRNA sites located in the coding region [4].

\section{miRNA:target interactions: a highly complex molecular network}

The development of bioinformatics tools has been a crucial step in the understanding of miRNA function either at a cellular or whole-organism level. Notably, it was shown that one miRNA could recognize tens of different mRNAs [5] and that one single mRNA could itself be the target of several miRNAs [6-8]. The corollary is that the longer the $3^{\prime}-U T R$ region of a mRNA, the higher the probability that it will be regulated by one or more miRNAs. It is also estimated that $60 \%$ of coding RNAs would be under the control of at least one miRNA. As a consequence, almost all biological processes would be directly or indirectly under the influence and control of miRNAs. Since the last inventory reported 2654 human miRNAs [9], the molecular landscape made of the biological interconnections between miRNAs and their target mRNAs within a cell is therefore particularly complex [5-10] and reminiscent of the neural interconnection network in the brain with its multiple nodes, areas, paths and branches. However, it is important to note that the robustness and accuracy of these predictions have often been questioned [3-11]. Only rigorous experimental approaches, including the use of reporter genes and site-directed mutagenesis $[6,7]$, can formally demonstrate the ability of a given miRNA to regulate a gene at the post-transcriptional level and identify the site of interaction between the miRNA and its target.

\section{Circulating microRNAs and exosomes}

miRNAs have also the peculiarity of being circulating in biological fluids such as blood, urine, tears or saliva (Figure 1). Circulating miRNAs are transported not as strands of bare RNA but associated with chaperone proteins, in particular Argonaut proteins, and lipoproteins, or wrapped in microvesicles (exosomes, microparticles, apoptotic bodies) that protect them from RNase degradation. These observations and the results of various studies show that the effector complex miRISC can be transported without damages by biological fluids, such as blood, and therefore exported from one part of the body to another, so that a miRNA can act at distance from its production site. This mechanism, known as transcommunication and associated with exosomes, opens up new perspectives in therapy, particularly in the field of RNA vectorization. However, some people believe that given the low number of miRISC per exosome, the physiological and pathophysiological impact of this phenomenon would be marginal [2].

\section{MicroRNAs, cancer and therapy}

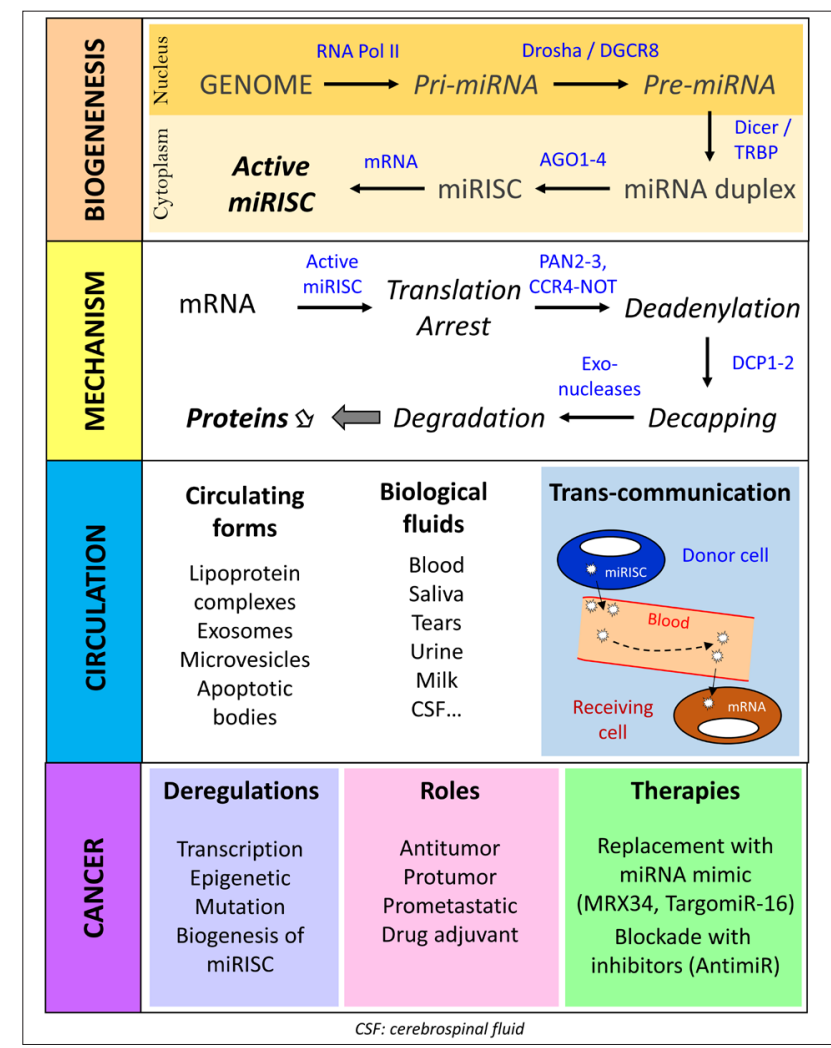

Figure 1: Biogenesis and biological and pathophysiological role of miRNA in cancer

In the light of the central role of miRNAs in gene regulation and biological processes, it was expected that the deregulation of their production would be closely linked to the development of cancers (Figure 1). Today, the query of Pubmed database with the keywords "microRNA" and "cancer" returns nearly 40,000 publications, pointing out the tremendous interest generated in cancerology by these small RNAs since their discovery. Following the first description in 2002 of the deregulation of miR-15 and miR-16 in 
chronic myeloid leukemia [12], many strategies have tempted to take advantage of the ability of miRNAs to regulate multiple genes simultaneously with the hope of increasing the efficacy of drug treatments while limiting their toxicity. The main challenge faced by developers is to specifically address the therapeutic miRNA to the tumor via the bloodstream, facilitate its entry into malignant cells, and finally act on its molecular targets with sufficient efficacy to achieve an antitumor effect. Two manufacturers have taken up this therapeutic challenge. The first Phase I clinical trial using a synthetic miR-34a-5p encapsulated in a liposomal shell (also known as MIRX34) was tested in the treatment of liver cancer, but this trial was hastily stopped due to major immune-related side effects [13]. On the other hand, a treatment using a synthetic miR-16 encapsulated in a bacterial vector (or TargomiR) has been successful and is currently in phase II clinical trial for the treatment of malignant pleural mesothelioma [14]. These latter results are particularly encouraging and underline the need to actively pursue research in the field of small RNA vectorization in order to improve transportation vehicles, such as those derived from exosomes.

\section{Conclusion}

Since their discovery in the 1990s, miRNAs generated considerable interest and expectation, especially between 2000 and 2015 . The tens of thousands of studies undertaken have led to a better understanding of their mode of action and a clear demonstration of their involvement in human pathology. The development of vectorization systems for small RNAs led to several clinical trials in 2014 in the treatment of cancers, one of which reached Phase II. Today, the specific targeting of these small RNAs in cancer cells remains a major challenge in therapy and oncology. It is likely that in a near future, vectorized forms of miRNA will become an integral part of the pharmaceutical arsenal used to treat patients presenting with cancer.

\section{Acknowledgements and Declaration of Interests}

We are grateful to the Dordogne, Gironde and Landes Committees of the 'Regional League against Cancer', the 'National Cancer Institute' (Grants INCa_PLBIO_2012_110 and INCa_ TRANSLA_2013_209), the 'Foundation for Medical Research' (Grant $\mathrm{n}^{\circ}$ DBI20131228566), the 'Ministry of Health' and the 'DirectorateGeneral for Caregiving' (Grant ${ }^{\circ}$ DGOS/PF4/0013/105), the French Government for its "Investments for the future" a program led by the University of Bordeaux (Post-doc IdEx program, ANR-10-
IDEX-03-02 grant), the 'Région Nouvelle-Aquitaine', the 'Groupama Foundation' and all patient associations (Aidons Marina, Cassandra, ESCAPE, Eva For Life, Kaëna and the Rabbits, The Flavian Foundation, The Friends of Marius, The Solidarity Motorcyclists, Spheres) for their support.

\section{References}

1. Jonas S, Izaurralde E (2015) Towards a molecular understanding of microRNA-mediated gene silencing. Nat Rev Genet 16(7): 421-433.

2. Gebert LFR, MacRae IJ (2019) Regulation of microRNA function in animals. Nat Rev Mol Cell Biol 20(1): 21-37.

3. Jalvy-Delvaille $\mathrm{S}$, et al (2012) Molecular basis of differential target regulation by miR-96 and miR-182: The glypican- 3 as a model. Nucleic Acids Res 40(3): 1356-1365.

4. Zhang K, et al (2018) A novel class of microRNA-recognition elements that function only within open reading frames. Nat Struct Mol Biol 25(11): 1019-1027.

5. Helwak A, Kudla G, Dudnakova T, Tollervey D (2013) Mapping the human miRNA interactome by CLASH reveals frequent noncanonical binding. Cell 153(3): 654-665.

6. Indersie E, et al (2017) MicroRNA therapy inhibits hepatoblastoma growth in vivo by targeting beta-catenin and wnt signaling. Hepatol Commun 1(2): 168-183.

7. Cartier F, et al (2017) New tumor suppressor microRNAs target glypican-3 in human liver cancer. Oncotarget 8(25): 41211-41226.

8. Maurel M, et al (2013) A functional screening identifies five micrornas controlling glypican-3: role of mir-1271 down-regulation in hepatocellular carcinoma. Hepatology 57(1): 195-204.

9. Kozomara A, Birgaoanu M, Griffiths-Jones S (2019) MiRBase: from microRNA sequences to function. Nucleic Acids Res 47(D1): D155-D162.

10. Vila-Casadesus M, et al (2018) Deciphering microRNA targets in pancreatic cancer using miRComb R package. Oncotarget 9(5): 64996517.

11. Pinzon N, et al (2017) MicroRNA target prediction programs predict many false positives. Genome Res 27(2): 234-245.

12. Calin GA, et al (2002) Frequent deletions and down-regulation of microRNA genes miR15 and miR16 at 13q14 in chronic lymphocytic leukemia. Proc Natl Acad Sci U S A 99(24): 15524-15529.

13. Beg MS, et al (2017) Phase I study of MRX34, a liposomal miR-34a mimic, administered twice weekly in patients with advanced solid tumors. Invest New Drugs 35(2): 180-188.

14. van Zandwijk NV, et al (2017) Safety and activity of microRNA-loaded minicells in patients with recurrent malignant pleural mesothelioma: a first-in-man, phase 1, open-label, dose-escalation study. Lancet Oncol 18(10): 1386-1396. 